\title{
Average Dwell-Time Bounds for ISS and Integral ISS of Switched Systems using Lyapunov Functions
}

\author{
Shenyu Liu
}

\author{
Aneel Tanwani
}

\begin{abstract}
The problem of input-to-state stability (ISS), and its integral version (iISS), is considered for switched systems with inputs and resets. The individual subsystems are assumed to be ISS (resp. iISS) with nonlinear decay rates in dissipation inequalities associated with the Lyapunov function of each subsystem. The change in the value of Lyapunov functions at switching instants is described by a nonlinear growth function. A generalized lower bound is computed for average dwell-time (ADT) to guarantee ISS/iISS of the switched system. In particular, an explicit formula of ADT lower bound is given for switched bilinear systems with zero-input-stable subsystems.
\end{abstract}

\section{INTRODUCTION}

Among the tools that have been developed for stability analysis of switched systems, the notion of dwell-time or average dwell-time (ADT) has played a significant role in characterization of stability conditions [1], [2]. In autonomous switched systems, a common recipe for computing such bounds is to associate a Lyapunov function with individual subsystems. Assuming exponential decay in the value of Lyapunov function during flows, and exponential growth at switching instants, lower bounds on ADT can be computed as a function of these decay and growth rates which guarantee asymptotic stability for that particular class of slowly switching signals.

For dynamical systems with inputs, the notion of input-to-state stability (ISS), or its close variant integral ISS (iISS), provides an elegant method to quantify the performance in control related applications. In the pioneering works on ISS [3] and iISS [4], these stability notions have also been characterized using Lyapunov functions. For switched systems, converse results regarding the existence of ISS and iISS systems first appeared in [5], and some implications relating ISS and iISS for switched systems have been developed in [6].

Combining the aforementioned two directions of research, it is rather natural to ask whether for slowly

S. Liu is with Department of Mechanical and Aerospace Engineering, University of California at San Diego, La Jolla, CA 92093, USA. A. Tanwani is with LAAS-CNRS, Université de Toulouse, CNRS, 31400 Toulouse, France. D. Liberzon is with Coordinated Science Laboratory, Department of Electrical and Computer Engineering, University of Illinois at Urbana-Champaign, Urbana IL 61801, USA.

S. Liu and D. Liberzon were supported by the NSF grant CMMI1662708 and the AFOSR grant FA9550-17-1-0236. A. Tanwani was supported by ANR project CONVAN with grant number ANR-17CE40-0019-01. switching systems, these Lyapunov characterizations can be combined with ADT notions to obtain sufficient conditions for ISS/iISS of switched systems. This question has indeed been addressed in the literature in different settings, and it is also the central topic of this paper. Early work dealing with ADT bounds for switched systems using Lyapunov functions for individual systems appears in [7]. Just like for autonomous systems, a major restriction of this work is that both the dissipation rates in the inequalities associated with the derivative of Lyapunov functions and the changes in the value of the Lyapunov functions at switching instants are assumed to be linear. While the dissipation rates are relaxed to be nonlinear in the study of ISS/iISS of impulsive systems, which are similar to switched systems, growth in the value of Lyapunov functions at impulses are not allowed in [8]. Switching dynamics with reset maps can also be captured by the framework of hybrid systems [9] and ISS characterizations via Lyapunov functions for hybrid systems have been developed in [10], [11]. Relaxing certain assumptions in these works, along with some developments based on converse results, iISS characterizations via Lyapunov functions for hybrid systems appear in [12].

Continuing with the research on computing ADT lower bounds which render the switched system ISS or iISS, the most relevant results for this paper have recently appeared in [13]. Compared to the earlier works, [13] considers a family of subsystems where the corresponding Lyapunov functions do not necessarily admit an exponential decay during flows. Similarly, a nonlinear estimate is assumed for growth in the value of Lyapunov functions of the individual subsystems at switching times. In this article, the formula of ADT reported in [13] for ISS switched systems is extended to iISS switched systems under some mild assumptions and with some appropriate modifications.

We also examine an application of our results in the context of bilinear switched systems, where the flow dynamics are bilinear with respect to the disturbances. A logarithmic Lyapunov function for (nonswitching) bilinear systems has been proposed in [14]. The same Lyapunov function can be used to show that individual systems are iISS if the unforced systems are asymptotically stable. The growth in the value of Lyapunov function at switching instants is also described by a sublinear function and hence the inequalities do not satisfy the 
assumptions of exponential growth/decay which are often found in the literature for computing ADT bounds. However, using the results derived in this paper, we obtain lower bounds on ADT with logarithmic Lyapunov functions which result in the bilinear switched system being iISS.

\section{Preliminaries}

In this section, we introduce the basic notation required for introducing the class of switched systems studied in this paper, and the definitions of ISS, iISS of switched systems under slow switching.

A function $\alpha: \mathbb{R}_{\geqslant 0} \longrightarrow \mathbb{R}_{\geqslant 0}$ is said to be positive definite if it is continuous, $\alpha(0)=0$ and $\alpha(s)>0$ for all $s>0$. If $\alpha$ is also strictly increasing, then it is said to be of class $\mathcal{K}$. In addition if $\alpha$ is also unbounded, then it is said to be of class $\mathcal{K}_{\infty}$. A function $\beta: \mathbb{R}_{\geqslant 0} \times \mathbb{R}_{\geqslant 0} \longrightarrow$ $\mathbb{R}_{\geqslant 0}$ is said to be of class $\mathcal{K} \mathcal{L}$ if $\beta(\cdot, t)$ is of class $\mathcal{K}$ for each $t \in \mathbb{R}_{\geqslant 0}, \beta(s, \cdot)$ is decreasing and $\beta(s, t) \longrightarrow 0$ as $t \longrightarrow \infty$ for each fixed $s \in \mathbb{R}_{\geqslant 0}$; see [15, Chapter 4$]$ for their use in formulation of common stability notions. In addition, we require class $\mathcal{K} \mathcal{L} \mathcal{L}$ functions: $\mathrm{A}$ function $\beta: \mathbb{R}_{\geqslant 0} \times \mathbb{R}_{\geqslant 0} \times \mathbb{R}_{\geqslant 0} \longrightarrow \mathbb{R}_{\geqslant 0}$ is a class $\mathcal{K} \mathcal{L} \mathcal{L}$ function if $\beta(\cdot, \cdot, j)$ is a class $\mathcal{K} \mathcal{L}$ function for each $j \geqslant 0$ and $\beta(\cdot, t, \cdot)$ is a class $\mathcal{K} \mathcal{L}$ function for each $t \geqslant 0$.

Let $\mathrm{P} \subset \mathbb{N}$ be a set of either finite or infinite cardinality. For each $p \in \mathrm{P}$, there is a locally Lipschitz vector field $f_{p}: \mathbb{R}^{n} \times \mathbb{R}^{m} \longrightarrow \mathbb{R}^{n}$. Moreover, these vector fields have the common equilibrium property, that is, $f_{p}(0,0)=0$, for each $p \in \mathrm{P}$. The differential equations

$$
\dot{x}=f_{p}(x, \omega), \quad p \in \mathrm{P}
$$

are the dynamics of the subsystems or modes of the switched system. For each pair $(p, q) \in \mathrm{P} \times \mathrm{P}$, there is also a jump map $g_{p, q}: \mathbb{R}^{n} \times \mathbb{R}^{m} \longrightarrow \mathbb{R}^{n}$ with the common equilibrium property that $g_{p, q}(0,0)=0$. Let $\bar{\Sigma}$ be the set of all right-continuous mappings from $\mathbb{R}_{\geqslant 0}$ to $\mathrm{P}$ with a locally finite number of discontinuities, called switching signals. For each switching signal $\sigma \in \bar{\Sigma}$, define $\mathcal{T}(\sigma):=\left\{t>0: \sigma(t) \neq \sigma\left(t^{-}\right)\right\}$; in other words, $\mathcal{T}(\sigma)$ is the collection of times when switches occur. With this data, we consider switched dynamical systems with inputs, described by,

$$
\begin{array}{r}
\dot{x}(t)=f_{\sigma(t)}\left(x\left(t^{-}\right), \omega(t)\right) \text { if } t \notin \mathcal{T}(\sigma), \\
x(t)=g_{\sigma(t), \sigma\left(t^{-}\right)}\left(x\left(t^{-}\right), \omega(t)\right) \text { if } t \in \mathcal{T}(\sigma),
\end{array}
$$

where $\omega: \mathbb{R}_{\geqslant 0} \longrightarrow \mathbb{R}^{m}$ is locally essentially bounded on $\mathbb{R}_{\geqslant 0}$ and bounded on $\mathcal{T}(\sigma)$. We denote the solution of (2) with initial state $x_{0}$, input $\omega$ and switching signal $\sigma$ by $x\left(\cdot ; x_{0}, \omega, \sigma\right)$. When $x_{0}, \omega, \sigma$ are clear from the context, the solution is also abbreviated by $x(\cdot)$. Note by definition of $(2), x\left(\cdot ; x_{0}, \omega, \sigma\right)$ obeys some differential equation when there is no switch, and it jumps when there is a switch.
For each $\sigma \in \bar{\Sigma}, N_{\sigma}(s, t)$ denotes the number of switches of $\sigma$ over the time interval $[s, t)$. A switching signal $\sigma$ admits an average dwell time (ADT) of $\tau_{a}>0$ if $\sigma \in \Sigma_{\tau_{a}}$, where

$$
\begin{array}{r}
\Sigma_{\tau_{a}}:=\left\{\sigma \in \bar{\Sigma}: \exists N_{0} \in \mathbb{N} \text { s.t } N_{\sigma}(s, t) \leqslant N_{0}+\frac{t-s}{\tau_{a}}\right. \\
\forall t>s \geqslant 0\} .
\end{array}
$$

Definition 1. Switched system (2) is uniformly inputto-state stable (ISS) over $\Sigma_{\tau_{a}}$ if there exist $\beta \in \mathcal{K} \mathcal{L}, \gamma \in \mathcal{K}$ such that

$$
\left|x\left(t ; x_{0}, \omega, \sigma\right)\right| \leqslant \beta\left(\left|x_{0}\right|, t\right)+\gamma\left(\operatorname{essup}_{s \in[0, t]}|\omega(s)|\right)
$$

for all $t \geqslant 0, x_{0} \in \mathbb{R}^{n}$ and $\sigma \in \Sigma_{\tau_{a}}$.

Definition 2. Switched system (2) is uniformly integral input-to-state stable (iISS) over $\Sigma_{\tau_{a}}$ if there exist $\alpha_{0} \in$ $\mathcal{K}_{\infty}, \beta \in \mathcal{K} \mathcal{L}, \gamma \in \mathcal{K}$ such that

$$
\alpha_{0}\left(\left|x\left(t ; x_{0}, \omega, \sigma\right)\right|\right) \leqslant \beta\left(\left|x_{0}\right|, t\right)+\int_{0}^{t} \gamma(|\omega(s)|) d s
$$

for all $t \geqslant 0, x_{0} \in \mathbb{R}^{n}$, and $\sigma \in \Sigma_{\tau_{a}}$.

Remark II.1. In [8], iISS for impulsive systems is defined via the following inequality

$$
\begin{aligned}
\alpha_{0}(|x(t)|) \leqslant \beta\left(\left|x_{0}\right|, t\right)+\int_{0}^{t} \gamma(|\omega(s)|) d s & \\
& +\sum_{t_{k} \in \mathcal{T}(\sigma)} \gamma\left(\left|\omega\left(t_{k}\right)\right|\right)
\end{aligned}
$$

where the effects of impulses are captured by the last summation term. The estimate in (5) is indeed a stronger version of the more general iISS definition in (6), and in this article we only develop conditions which lead to iISS in the sense of (5). Our proposed conditions for establishing iISS, as in (5), are therefore stronger than one would expect in general for the estimate (6). This can indeed be seen from the upcoming assumption (L3) that the jump dynamics is independent of input disturbance.

\section{Assumptions And Main Result}

We now state some assumptions on the data of system (2) required for the statement of the main result.

Assumption 1. There exist $\mathcal{C}^{1}$ Lyapunov functions $V_{p}$ : $\mathbb{R}^{n} \longrightarrow \mathbb{R}_{\geqslant 0}$, satisfying the conditions:

(L1) There exist $\underline{\alpha}, \bar{\alpha} \in \mathcal{K}_{\infty}$ such that

$$
\underline{\alpha}(|x|) \leqslant V_{p}(x) \leqslant \bar{\alpha}(|x|), \quad \forall x \in \mathbb{R}^{n}, p \in \mathrm{P} .
$$

(L2) There exist a positive definite function $\alpha$ and $\gamma \in$ $\mathcal{K}$ such that

$$
\left\langle\frac{\partial}{\partial x} V_{p}(x), f_{p}(x, \omega)\right\rangle \leqslant-\alpha\left(V_{p}(x)\right)+\gamma(|\omega|) .
$$

for all $x \in \mathbb{R}^{n}, \omega \in \mathbb{R}^{m}$. 
(L3) There exists $\chi \in \mathcal{K}_{\infty}$, such that for each $(p, q) \in$ $\mathrm{P} \times \mathrm{P}$

$$
V_{q}\left(g_{p, q}(x, \omega)\right) \leqslant \chi\left(V_{p}(x)\right), \quad \forall x \in \mathbb{R}^{n}, \omega \in \mathbb{R}^{m} .
$$

Remark III.1. For each subsystem in (1), (L1) and (L2) provide a necessary and sufficient condition for the subsystem to be iISS [16]. Uniform bounds $\underline{\alpha}, \bar{\alpha}$ in (L1) exist when $\mathrm{P}$ has a finite cardinality or when $V_{p}$ are uniformly bounded with respect to $p \in \mathrm{P}$. In addition, if it is assumed that $\alpha$ in (L2) is $\mathcal{K}_{\infty}$, then each subsystem described by (1) is ISS.

Based on Assumption 1, we introduce the function $\psi$ : $\mathbb{R}_{\geqslant 0} \longrightarrow \mathbb{R}_{\geqslant 0}$ as

$$
\psi(r):=\min \{\alpha(r), c r\}
$$

where $c>0$ is some constant. The next assumption that we need relates to the regularity of the function $\psi$.

Assumption 2. The function $\psi$ is globally one-sided Lipschitz with constant $c_{0} \geqslant 0$, in the sense that, for any $w \geqslant v \geqslant 0, \psi(w)-\psi(v) \leqslant c_{0}(w-v)$.

The last assumption is adopted from [13]. It provides a lower bound on ADT such that the switched system is ISS/iISS.

Assumption 3. The supremum

$$
\zeta^{*}:=\sup _{s>0} \int_{s}^{\chi(s)} \frac{1}{\psi(r)} d r
$$

is finite.

It is noticed that for linear systems and bilinear systems, proper choices of the Lyapunov functions do give finite value of $\zeta^{*}$. However, it is also noticed that the nonlinear switched system in [17, Section 3] does not have a finite $\zeta^{*}$. Indeed, that system is not iISS no matter how slowly it switches; it is only quasi-iISS under slow switching in the sense defined in [17].

Now we are ready to present our main result:

Theorem III.2. Consider the switched system (2) and suppose that Assumptions 1-3 hold. Then the system (2) is uniformly iISS over $\Sigma_{\tau_{a}}$ for any $\tau_{a}>\zeta^{*}$. In addition, if in (L2) $\alpha \in \mathcal{K}_{\infty}$, then the system (2) is uniformly ISS over $\Sigma_{\tau_{a}}$ for any $\tau_{a}>\zeta^{*}$.

\section{Integral ISS of Bilinear Systems}

In this section, we demonstrate how Theorem III.2 can be used to find a lower bound on the ADT for the switched system with bilinear subsystems

$$
\begin{aligned}
\dot{x} & =f_{p}(x, \omega)=A_{p} x+\sum_{j=1}^{m} B_{p, j} x \omega_{j}+C_{p} \omega, \\
x^{+} & =x
\end{aligned}
$$

to be iISS. For this system (12) we assume $p \in \mathrm{P}=$ $\{1,2, \cdots P\}, x \in \mathbb{R}^{n}, \omega=\left(\begin{array}{lll}\omega_{1} & \cdots & \omega_{m}\end{array}\right)^{\top} \in \mathbb{R}^{m}$ and $A_{p} \in \mathbb{R}^{n \times n}$ are all Hurwitz, $B_{p, j} \in \mathbb{R}^{n \times n}$ and $C_{p} \in$ $\mathbb{R}^{n \times m}$. It is known from [4] that each bilinear subsystem, with $A_{p}$ being Hurwitz, is iISS. In particular, this is enough to guarantee that Assumption 1 holds.

\section{A. Assumption 1 holds if each $A_{p}$ is Hurwitz}

Indeed, for each $p \in \mathbf{P}$, we can consider the iISSLyapunov function $V_{p}(x)$ as (see, e.g., [14])

$$
V_{p}(x):=\ln \left(1+x^{\top} M_{p} x\right)
$$

where $M_{p}$ is a symmetric positive definite matrix that satisfies

$$
M_{p} A_{p}+A_{p}^{\top} M_{p}+Q_{p}=0
$$

for some symmetric positive definite matrices $Q_{p} \in$ $\mathbb{R}^{n \times n}$. Item (L1) in Assumption 1 is satisfied with $\underline{\alpha}(s)=$ $\ln \left(1+\underline{a} s^{2}\right), \bar{\alpha}(s)=\ln \left(1+\bar{a} s^{2}\right)$ where

$$
\bar{a}=\max _{p \in \mathrm{P}}\left|\bar{\lambda}\left(M_{p}\right)\right|, \quad \underline{a}=\min _{p \in \mathrm{P}}\left|\underline{\lambda}\left(M_{p}\right)\right|
$$

and $\bar{\lambda}(\cdot)$ and $\underline{\lambda}(\cdot)$ denote the maximum and minimum eigenvalues respectively. Next, to show that (L2) hold, it can be algebraically verified that

$$
\begin{aligned}
& \left\langle\frac{d}{d x} V_{p}(x), f_{p}(x, \omega)\right\rangle \\
& \leqslant-\frac{b|x|^{2}}{1+x^{\top} M_{p} x}+\frac{c_{1}|x|^{2}+c_{2}|x|}{1+x^{\top} M_{p} x}|\omega| \\
& \leqslant-\frac{b\left(e^{V_{p}(x)}-1\right)}{\bar{a} e^{V_{p}(x)}}+\left(\frac{c_{1}}{\underline{a}}+\frac{c_{2}}{2 \sqrt{\underline{a}}}\right)|\omega|
\end{aligned}
$$

for all $p \in \mathrm{P}$, where $b$ is defined by

$$
b:=\min _{p \in \mathrm{P}}\left|\underline{\lambda}\left(Q_{p}\right)\right|,
$$

and $c_{1}, c_{2}$ are given by

$$
\begin{aligned}
c_{1}: & =\sqrt{m} \max _{j \in\{1, \ldots, m\}, p \in \mathrm{P}}\left|\bar{\lambda}\left(B_{p, j}^{\top} M_{p}+M_{p} B_{p, j}\right)\right|, \\
c_{2} & :=2 \max _{p \in \mathrm{P}}\left|\bar{\lambda}\left(M_{p} C_{p}\right)\right| .
\end{aligned}
$$

Thus by setting $\alpha(s) \quad:=\frac{b\left(e^{s}-1\right)}{\bar{a} e^{s}}$ and $\gamma(s) \quad:=$ $\left(\frac{c_{1}}{\underline{a}}+\frac{c_{2}}{2 \sqrt{\underline{a}}}\right) s$, we conclude (8). In addition, by definition we see that $\alpha$ is positive definite and $\gamma \in \mathcal{K}$ so that item (L2) in Assumption 1 is verified: each subsystem of (12) is iISS.

Finally, we check (L3) and compute the map $\chi$ in (9). To do so, let us introduce a constant $\mu \geqslant 1$ such that

$$
x^{\top} M_{q} x \leqslant \mu x^{\top} M_{p} x
$$

for every $p, q \in \mathrm{P}$ and $x \in \mathbb{R}^{n}$. Consequently, we get

$$
\begin{aligned}
V_{q}(x) & =\ln \left(1+x^{\top} M_{q} x\right) \leqslant \ln \left(1+\mu x^{\top} M_{p} x\right) \\
& =\ln \left(1+\mu\left(e^{V_{p}(x)}-1\right)\right) .
\end{aligned}
$$

We conclude that (9) holds with

$$
\chi(s)=\ln \left(\mu\left(e^{s}-1\right)+1\right) .
$$




\section{B. Assumption 2, Assumption 3 and ADT bound}

Next, we construct the map $\psi$ as defined in (10), and compute $\zeta^{*}$ in (11). Notice that $\alpha^{\prime}(s)=\frac{b}{\bar{a} e^{s}} \leqslant \frac{b}{\bar{a}}$ for all $s \geqslant 0$, we can pick $c=\frac{b}{\bar{a}}$ such that $\psi(s)=\alpha(s)=$ $\frac{b\left(e^{s}-1\right)}{\bar{a} e^{s}}$. Using (11), the lower bound on ADT is

$$
\begin{aligned}
\zeta^{*} & =\sup _{s>0} \int_{s}^{\chi(s)} \frac{1}{\psi(r)} d r=\sup _{s>0} \int_{s}^{\chi(s)} \frac{\bar{a} e^{r}}{b\left(e^{r}-1\right)} d r \\
& =\left.\sup _{s>0} \frac{\bar{a}}{b} \ln \left(e^{r}-1\right)\right|_{s} ^{\ln \left(\mu\left(e^{s}-1\right)+1\right)} \\
& =\sup _{s>0} \frac{\bar{a}}{b} \ln \frac{\left(\mu\left(e^{s}-1\right)+1\right)-1}{e^{s}-1} \\
& =\frac{\bar{a}}{b} \ln \mu .
\end{aligned}
$$

From Theorem III.2, it holds that the switched bilinear system (12) is iISS for each $\sigma \in \Sigma_{\tau_{a}}$, when

$$
\tau_{a}>\frac{\bar{a}}{b} \ln \mu \text {. }
$$

An immediate observation resulting from the foregoing expression is that, when there exists a common Lyapunov function, all $M_{p}$ 's are the same and we can choose $\mu=1$ which results in the lower bound on ADT in (19) being 0 . This is indeed the case because the switched system is iISS with arbitrary switching [5].

\section{Technical Tools and Proofs}

As mentioned in the introduction, our results of ISS/iISS switched system rely on rewriting the switched system into a hybrid system in the framework of [9] and then showing ISS/iISS of that hybrid system. In this section we provide a brief description of hybrid systems, followed with some supporting lemmas used in the proof of Theorem III.2.

\section{A. Modeling as hybrid systems}

Consider the hybrid dynamical system with inputs, which is described as:

$$
\left\{\begin{array}{cl}
\dot{\xi} \in \mathcal{F}(\xi, d) & (\xi, d) \in \mathcal{C} \\
\xi^{+} \in \mathcal{G}(\xi, d) & (\xi, d) \in \mathcal{D}
\end{array}\right.
$$

where the state trajectory $\xi$ evolves in $\mathcal{X}$, and the disturbance $d$ takes values in $\mathbb{R}^{m}$. The sets $\mathcal{C}, \mathcal{D}$ are subsets of $\mathcal{X} \times \mathbb{R}^{m}$ and are called flow, and jump sets, respectively. The evolution of the state is thus described by $\mathcal{F}$ (during flows) and by $\mathcal{G}$ (at jump instants), which are set-valued mappings from $\mathcal{X} \times \mathbb{R}^{m}$ to $\mathcal{X}$. It is assumed that the system data $(\mathcal{F}, \mathcal{G}, \mathcal{C}, \mathcal{D})$ satisfies certain basic assumptions [9], [11] such that, for each input $d: \operatorname{dom} d \longrightarrow \mathbb{R}^{m}$, the system (20) admits a local solution, called hybrid arc, $\xi: \operatorname{dom} \xi \longrightarrow \mathcal{X}$, where $\operatorname{dom} \xi \subset \mathbb{R} \times \mathbb{N}$. For an initial state $\xi_{0}$ and an input $d$, a hybrid arc of (20) is denoted by $\xi\left(\cdot, \cdot ; \xi_{0}, d\right)$. In what follows, we denote the distance between a vector $\xi \in \mathcal{X}$ and a compact set $\mathcal{A} \subset \mathcal{X}$ by $|\xi|_{\mathcal{A}}$, that is, $|\xi|:=\inf _{\zeta \in \mathcal{A}}|\xi-\zeta|$. Following [10], for a hybrid signal $d$, we use the notation $\|d\|_{(t, j)}$ to denote the maximum between ess sup $|d(\hat{t}, \hat{j})|$ and $\sup _{(\hat{t}, \hat{j}+1) \in \operatorname{dom} d, \hat{t}+\hat{j} \leqslant t+j}|d(\hat{t}, \hat{j})|$.

Definition 3. A hybrid system (20) is said to be inputto-state stable (ISS) with respect to $\mathcal{A}$ if there exists $\beta \in$ $\mathcal{K} \mathcal{L} \mathcal{L}$ and $\gamma \in \mathcal{K}$ such that for all $\xi_{0} \in \mathcal{X}$ and all $(t, j) \in$ $\operatorname{dom} \xi$, each solution pair $(\xi, d)$ satisfies

$$
\left|\xi\left(t, j ; \xi_{0}, d\right)\right|_{\mathcal{A}} \leqslant \beta\left(\left|\xi_{0}\right|_{\mathcal{A}}, t, j\right)+\gamma\left(\|d\|_{(t, j)}\right) .
$$

Definition 4. A hybrid system (20) is said to be integral input-to-state stable (iISS) with respect to $\mathcal{A}$ if there exists $\alpha \in \mathcal{K}_{\infty}, \beta \in \mathcal{K} \mathcal{L} \mathcal{L}$ and $\gamma \in \mathcal{K}$ such that for all $\xi_{0} \in \mathcal{X}$ and all $(t, j) \in \operatorname{dom} \xi$, each solution pair $(\xi, d)$ satisfies

$$
\alpha\left(\left|\xi\left(t, j ; \xi_{0}, d\right)\right|_{\mathcal{A}}\right) \leqslant \beta\left(\left|\xi_{0}\right|_{\mathcal{A}}, t, j\right)+\int_{0}^{t} \gamma\left(\left|d\left(s, j_{d, s}\right)\right|\right) d s
$$

where $j_{d, s}:=\max \{i \in \mathbb{N} \mid(s, i) \in \operatorname{dom} d\}$.

We can also characterize ISS and iISS using Lyapunov functions, as stated in the following two lemmas [10], [12]:

Lemma V.1. A hybrid system (20) is ISS with respect to $\mathcal{A}$ if there exists a smooth function $V: \mathcal{X} \longrightarrow \mathbb{R}_{\geqslant 0}$ and functions $\alpha_{1}, \alpha_{2}, \alpha_{c}, \alpha_{d} \in \mathcal{K}_{\infty}$ and $\gamma_{c} \in \mathcal{K}$ such that

$$
\alpha_{1}\left(|\xi|_{\mathcal{A}}\right) \leqslant V(\xi) \leqslant \alpha_{2}\left(|\xi|_{\mathcal{A}}\right)
$$

for all $\xi \in \mathcal{X}$

$$
\left\langle\frac{\partial}{\partial \xi} V(\xi), \bar{f}\right\rangle \leqslant-\alpha_{c}\left(|\xi|_{\mathcal{A}}\right)+\gamma_{c}(|d|)
$$

for all $(\xi, d) \in \mathcal{C}, \bar{f} \in \mathcal{F}(\xi, d)$, and

$$
V(\bar{g})-V(\xi) \leqslant-\alpha_{d}\left(|\xi|_{\mathcal{A}}\right)
$$

for all $(\xi, d) \in \mathcal{D}, \bar{g} \in \mathcal{G}(\xi, d)$.

Lemma V.2. A hybrid system is iISS with respect to $\mathcal{A}$ if there exists a smooth function $V: \mathcal{X} \longrightarrow \mathbb{R}_{\geqslant 0}$ and functions $\alpha_{1}, \alpha_{2} \in \mathcal{K}_{\infty}$, positive definite functions $\alpha_{c}, \alpha_{d}$ and $\gamma_{c} \in \mathcal{K}$ such that (23) holds for all $\xi \in \mathcal{X},(24)$ holds for all $(\xi, d) \in \mathcal{C}, \bar{f} \in \mathcal{F}(\xi, d)$ and (25) holds for all $(\xi, d) \in \mathcal{D}, \bar{g} \in \mathcal{G}(\xi, d)$.

We call a $V$ satisfying the inequalities in Lemma $\mathrm{V} .1$ (resp. Lemma V.2) a hybrid ISS (resp. iISS) Lyapunov function.

Remark V.3. Note that Lemma V.2 differs from Lemma V.1 in the sense that $\alpha_{c}, \alpha_{d}$ are only assumed to be positive definite instead of class $\mathcal{K}_{\infty}$, which also implies that iISS is strictly weaker than ISS.

\section{B. ADT switching and hybrid systems}

One can write the switched system (2), along with the ADT constraint on the switching signal, more naturally 
in the form of (20). To do so, we let

$$
\xi:=\left(\begin{array}{c}
x \\
p \\
\tau
\end{array}\right) \in \mathbb{R}^{n} \times \mathrm{P} \times\left[0, N_{0}\right]=: \mathcal{X} .
$$

The hybrid model capturing the dynamics of the switched system, driven by an $\mathbb{R}^{m}$-valued disturbance $d$, and a switching signal $\sigma \in \Sigma_{\tau_{a}}$, is compactly written as

$$
\begin{gathered}
\dot{\xi} \in \mathcal{F}(\xi, d):\left\{\begin{array}{l}
\dot{x}=f_{p}(x, d) \\
\dot{p}=0 \\
\dot{\tau} \in\left[0, \frac{1}{\tau_{a}}\right]
\end{array} \quad \text { if } \quad(\xi, d) \in \mathcal{C}\right. \\
\xi^{+} \in \mathcal{G}(\xi, d):\left\{\begin{array}{l}
x^{+} \in G_{p}(x, d) \\
p^{+} \in \mathrm{P} \\
\tau^{+}=\tau-1
\end{array} \text { if }(\xi, d) \in \mathcal{D}\right.
\end{gathered}
$$

where $\mathcal{C}=\mathcal{X} \times \mathbb{R}^{m}, \mathcal{D}=\mathbb{R}^{n} \times \mathrm{P} \times\left[1, N_{0}\right] \times \mathbb{R}^{m}$, and $G_{p}(x, d)=\bigcup_{q \in \mathrm{P}} g_{p, q}(x, d)$. The attractor of the system (27) is the compact set

$$
\mathcal{A}:=\{0\}^{n} \times \mathrm{P} \times\left[0, N_{0}\right] .
$$

A similar modeling of a switched system with ADT constraint in hybrid system framework can also be found in [18]. Comparing (21) and (22) with (4) and (5) respectively, we have the following straightforward result:

Corollary V.4. The switched system (2) with its switching signal admitting ADT of $\tau_{a}$ is ISS (resp. iISS) if the hybrid system (27), with augmented state variable defined in (26), is ISS (resp. iISS) with respect to $\mathcal{A}$.

\section{Supporting Lemmas}

Define the function $\varphi: \mathbb{R}_{\geqslant 0} \longrightarrow \mathbb{R}_{\geqslant 0}$ by

$$
\varphi(s):=\left\{\begin{array}{cc}
\exp \left(\int_{1}^{s} \frac{2 c}{\psi(r)} d r\right) & s>0, \\
0 & s=0 .
\end{array}\right.
$$

where we recall that $c$ and $\psi$ are introduced in (10). We state two properties associated with $\psi$ and $\varphi$. Due to space constraints, the proofs are omitted and can be found in the online technical report [19].

Lemma V.5. It holds that $\varphi \in \mathcal{K}_{\infty}$.

Proof. It suffices to show that $\varphi$ is continuous at 0 and $\lim _{s \longrightarrow \infty} \varphi(s)=\infty$. Note that $\frac{2 c}{\psi(r)} \geqslant \frac{2}{r}$ and hence when $s>1, \int_{1}^{s} \frac{2 c}{\psi(r)} d r \geqslant \int_{1}^{s} \frac{2 c}{c r} d r=2 \ln s$ so $\lim _{s \longrightarrow \infty} \varphi(s) \geqslant$ $\lim _{s \rightarrow \infty} s^{2}=\infty$. On the other hand when $s<1$, $\int_{1}^{s} \frac{2 c}{\psi(r)} d r \leqslant \int_{1}^{s} \frac{2 c}{c r} d r=2 \ln s$ so $\lim _{s \longrightarrow 0^{+}} \varphi(s) \leqslant$ $\lim _{s \longrightarrow 0^{+}} s^{2}=0$ and thus $\varphi$ is continuous at 0 .

Lemma V.6. Let $c_{1}>0$ and $w \geqslant v>0$ such that $\varphi(w) \leqslant c_{1} \varphi(v)$. Then under Assumption 2, $\frac{\psi(w)}{\psi(v)} \leqslant c_{1}^{\frac{c}{2 c}}$.

Proof. Fix $w \geqslant v>0$. Then, $\varphi(w) \leqslant c_{1} \varphi(v)$ means $\exp \left(\int_{1}^{w} \frac{2 c}{\psi(r)} d r\right) \leqslant c_{1} \exp \left(\int_{1}^{v} \frac{2 c}{\psi(r)} d r\right)$. Take logarithm on both sides and subtract the integral on the right from the left, we get

$$
\int_{v}^{w} \frac{2 c}{\psi(r)} d r \leqslant \ln \left(c_{1}\right) .
$$

Define $\widetilde{\psi}_{v}:[v, \infty) \longrightarrow \mathbb{R}_{+}: \widetilde{\psi}_{v}(r)=\psi(v)+c_{0}(r-v)$. Recall that $c_{0}$ is the global one-sided Lipschitz constant of $\psi$, so by Assumption 2, we have $\widetilde{\psi}_{v}(r) \geqslant \psi(r)$ for all $r \geqslant v$. Hence, continuing from (30), we have

$$
\begin{aligned}
& \ln \left(c_{1}\right) \geqslant \int_{v}^{w} \frac{2 c}{\psi(r)} d r \geqslant \int_{v}^{w} \frac{2 c}{\widetilde{\psi}_{v}(r)} d r \\
&=\int_{\widetilde{\psi}_{v}(v)}^{\widetilde{\psi}_{v}(w)} \frac{2 c}{s} d\left(\widetilde{\psi}_{v}^{-1}(s)\right)=\frac{2 c}{c_{0}} \int_{\widetilde{\psi}_{v}(v)}^{\widetilde{\psi}_{v}(w)} \frac{1}{s} d s \\
& \quad=\frac{2 c}{c_{0}} \ln \frac{\widetilde{\psi}_{v}(w)}{\widetilde{\psi}_{v}(v)} \geqslant \frac{2 c}{c_{0}} \ln \frac{\psi(w)}{\psi(v)},
\end{aligned}
$$

which results in $\frac{\psi(w)}{\psi(v)} \leqslant c_{1}^{\frac{c_{0}}{2 c}}$.

Theorem III.2 is a direct consequence of Lemma V.1, Lemma V.2, Corollary V.4, and the following Lemma, which we prove in the remainder of this section.

Lemma V.7. Consider the hybrid system (27). Suppose Assumptions 1-3 hold and $\tau_{a}>\zeta^{*}$. For any $\zeta>0$ such that $\tau_{a}>\zeta>\zeta^{*}$,

$$
V(\xi):=\varphi^{-1}\left(e^{2 c \zeta \tau} \varphi\left(V_{p}(x)\right)\right)
$$

is a hybrid iISS Lyapunov function with respect to the compact set $\mathcal{A}$. In addition if we further assume that in (L2) $\alpha \in \mathcal{K}_{\infty}$, then (31) is an ISS Lyapunov function with respect to $\mathcal{A}$.

Proof. Before we start the proof, we note that $\varphi^{-1}$ in (31) is well-defined because $\varphi \in \mathcal{K}_{\infty}$, as stated in Lemma V.5.

To check the "sandwich" condition (23), we simply define

$\alpha_{1}(s):=\varphi^{-1}(\varphi(\underline{\alpha}(s))), \quad \alpha_{2}(s):=\varphi^{-1}\left(e^{2 c \zeta N_{0}} \varphi(\bar{\alpha}(s))\right)$.

We now want to show (24) and we only consider the non-trivial case when $x \neq 0$. Define

$$
W(\xi):=e^{2 c \zeta \tau} \varphi\left(V_{p}(x)\right) .
$$

Then $V(\xi)=\varphi^{-1}(W(\xi))$. Pick $(\xi, d) \in \mathcal{C}$ and $\bar{f} \in$ $\mathcal{F}(\xi, d)$, we have

$$
\begin{aligned}
& \left\langle\frac{\partial}{\partial \xi} W(\xi), \bar{f}\right\rangle \\
& \quad=2 c \zeta e^{2 c \zeta \tau} \varphi\left(V_{p}(x)\right) \dot{\tau}+\left\langle e^{2 c \zeta \tau} \varphi^{\prime}\left(V_{p}(x)\right) \frac{\partial}{\partial x} V_{p}(x), f_{p}\right\rangle \\
& \quad \leqslant \frac{2 c \zeta W(\xi)}{\tau_{a}}+\frac{2 c W(\xi)}{\psi\left(V_{p}(x)\right)}\left(-\alpha\left(V_{p}(x)\right)+\gamma(|d|)\right) \\
& \quad \leqslant 2 c\left(\frac{\zeta}{\tau_{a}}-1\right) W(\xi)+\frac{2 c W(\xi)}{\psi\left(V_{p}(x)\right)} \gamma(|d|)
\end{aligned}
$$


where we have used the fact that $\varphi^{\prime}(s)=\frac{2 c \varphi(s)}{\psi(s)}$ in the first inequality, and the property $\psi(s) \leqslant \alpha(s)$ for the second inequality above. Note that since we have picked $\zeta<\tau_{a}, \frac{\zeta}{\tau_{a}}<1$, it holds that $a:=\left(1-\frac{\zeta}{\tau_{a}}\right)>0$.

Next, to compute the derivative of $V$ along the differential inclusion, notice that $\left(\varphi^{-1}\right)^{\prime}(s)=\frac{1}{\varphi^{\prime}\left(\varphi^{-1}(s)\right)}=$ $\frac{\psi\left(\varphi^{-1}(s)\right)}{2 c s}$, which results in

$$
\begin{aligned}
& \left\langle\frac{\partial}{\partial \xi} V(\xi), \bar{f}\right\rangle=\left(\varphi^{-1}\right)^{\prime}(W(\xi))\left\langle\frac{\partial}{\partial \xi} W(\xi), \bar{f}\right\rangle \\
& \quad \leqslant\left(\frac{\psi\left(\varphi^{-1}(W(\xi))\right)}{2 c W(\xi)}\right)\left(-2 c a W(\xi)+\frac{2 c W(\xi)}{\psi\left(V_{p}(x)\right)} \gamma(|d|)\right) \\
& \quad=-a \psi\left(\varphi^{-1}(W(\xi))\right)+\frac{\psi\left(\varphi^{-1}(W(\xi))\right)}{\psi\left(V_{p}(x)\right)} \gamma(|d|) \\
& \quad=-a \psi(V(\xi))+\frac{\psi(V(\xi))}{\psi\left(V_{p}(x)\right)} \gamma(|d|) .
\end{aligned}
$$

To further simplify the right-hand side, let $v:=V_{p}(x)$, $w=V(\xi) ;$ then clearly $\varphi(w)=W(\xi)=e^{2 c \zeta \tau} \varphi\left(V_{p}(x)\right) \leqslant$ $e^{2 c \zeta N_{0}} \varphi(v)$, and from Lemma V.6,

$$
\begin{aligned}
\left\langle\frac{\partial}{\partial \xi} V(\xi), \bar{f}\right\rangle & \leqslant-a \psi(V(\xi))+\frac{\psi(V(\xi))}{\psi\left(V_{p}(x)\right)} \gamma(|d|) \\
& \leqslant-a \psi(V(\xi))+e^{c_{0} \zeta N_{0}} \gamma(|d|) \\
& \leqslant-a \psi \circ \alpha_{1}\left(|\xi|_{\mathcal{A}}\right)+e^{c_{0} \zeta N_{0}} \gamma(|d|)
\end{aligned}
$$

Hence, (24) holds with $\alpha_{c}(s)=a \psi \circ \alpha_{1}(s), \gamma_{c}(s)=$ $e^{c_{0} \zeta N_{0}} \gamma(s)$. It follows from the definition of $\psi$ in (10) that, if $\alpha$ is positive definite (resp. $\alpha \in \mathcal{K}_{\infty}$ ), then $\alpha_{c}$ is also positive definite (resp. $\alpha_{c} \in \mathcal{K}_{\infty}$ ). In addition, $\gamma_{c} \in \mathcal{K}$.

To show (25), pick $(\xi, d) \in \mathcal{D}$ and $\bar{g} \in \mathcal{G}(\xi, d)$. By the definition of $\varphi$ in (29) and $\zeta^{*}$ in (11) we have

$$
\begin{aligned}
& \varphi\left(\chi\left(V_{p}(x)\right)\right)=\exp \left(\int_{1}^{\chi\left(V_{p}(x)\right)} \frac{2 c}{\psi(r)} d r\right) \\
& =\exp \left(\int_{V_{p}(x)}^{\chi\left(V_{p}(x)\right)} \frac{2 c}{\psi(r)} d r\right) \exp \left(\int_{1}^{V_{p}(x)} \frac{2 c}{\psi(r)} d r\right) \\
& \leqslant e^{2 c \zeta^{*}} \varphi\left(V_{p}(x)\right) .
\end{aligned}
$$

Therefore,

$$
\begin{aligned}
V(\bar{g}) & =\varphi^{-1}\left(e^{2 c \zeta \tau^{+}} \varphi\left(V_{p^{+}}\left(x^{+}\right)\right)\right) \\
& \leqslant \varphi^{-1}\left(e^{2 c \zeta(\tau-1)} \varphi\left(\chi\left(V_{p}(x)\right)\right)\right) \\
& \leqslant \varphi^{-1}\left(e^{2 c \zeta(\tau-1)+2 c \zeta^{*}} \varphi\left(V_{p}(x)\right)\right) \\
& \leqslant \varphi^{-1}\left(e^{2 c\left(\zeta^{*}-\zeta\right)} \varphi(V(\xi))\right) .
\end{aligned}
$$

Define

$$
\kappa(v):=v-\varphi^{-1}\left(e^{2 c\left(\zeta^{*}-\zeta\right)} \varphi(v)\right),
$$

the inequality (25) is seen to hold with $\alpha_{d}(s)=\kappa \circ \alpha_{1}(s)$. Recall that $\zeta>\zeta^{*}$ and $\varphi \in \mathcal{K}_{\infty}$; thus by construction $\kappa$ is positive definite, so is $\alpha_{d}$. Lastly we show that $\alpha_{d} \in \mathcal{K}_{\infty}$ when $\alpha \in \mathcal{K}_{\infty}$ and it suffices to show that $\kappa \in \mathcal{K}_{\infty}$. It follows from $(33)$ that $\varphi\left((v-\kappa(v))=e^{2 c\left(\zeta^{*}-\zeta\right)} \varphi(v)\right.$ and, after plugging (29) in and taking logarithm, we get

$$
\int_{1}^{v-\kappa(v)} \frac{2 c}{\psi(r)} d r=2 c\left(\zeta^{*}-\zeta\right)+\int_{1}^{v} \frac{2 c}{\psi(r)} d r .
$$

Equivalently,

$$
\int_{v-\kappa(v)}^{v} \frac{1}{\psi(r)} d r=\zeta-\zeta^{*}>0 .
$$

When $\alpha \in \mathcal{K}_{\infty}, \frac{1}{\psi(r)}$ decreases to 0 as $r$ increases to infinity; therefore, (34) holds for all $v>0$ only when the interval of integration grows to infinity with respect to $v$. In other words, we conclude that $\kappa \in \mathcal{K}_{\infty}$.

As a final remark, we conclude from Lemma V.2 (resp. Lemma V.1) that $V$ is an iISS (resp. ISS) Lyapunov function.

\section{Conclusions}

Within the context of stability of switched systems under slow switching, this paper provides lower bounds on ADT which guarantee ISS or iISS properties. In particular, we considered the case where each subsystem is ISS (resp. iISS). Using the nonlinear supply functions associated with these subsystems, and the growth functions describing the change in the value of Lyapunov functions at switching instants, a lower bound on ADT is computed which guarantees ISS (resp. iISS) for a class of switching signals satisfying such a bound. It is seen that switched bilinear systems indeed fall within the framework studied in this paper, and we have provided conditions under which slowly switching bilinear systems are seen to be iISS. Among several possible extensions, it is desirable to adopt this framework under relaxed hypotheses which allow for destabilizing effect of disturbances in jump dynamics, and a wider class of supply functions associated with individual subsystems. Since iISS property has found utility in analyzing interconnections of dynamical systems [20], one can also use the results of this paper to analyse stability of interconnections of switched systems under slow switching.

\section{REFERENCES}

[1] J. Hespanha and A. Morse, "Stability of switched systems with average dwell-time," in Proceedings of 38th IEEE Conf. on Decision and Control, 1999, pp. 2655-2660.

[2] D. Liberzon, Switching in Systems and Control. Boston: Birkhäuser, 2003.

[3] E. D. Sontag, "Smooth stabilization implies coprime factorization," IEEE Trans. Automatic Control, vol. 34, no. 4, pp. 435 $-443,1989$.

[4] E. Sontag, "Comments on integral variants of ISS," Systems \& Control Letters, vol. 34, no. 1, pp. 93-100, 1998.

[5] J. Mancilla-Aguilar and R. García, "On converse Lyapunov theorems for ISS and iISS switched nonlinear systems," Systems $\& 3$ Control Letters, vol. 42, no. 1, pp. 47 - 53, 2001.

[6] H. Haimovich and J. Mancilla-Aguilar, "ISS implies iISS even for switched and time-varying systems (if you are careful enough)," Automatica, vol. 104, pp. 154 - 164, 2019. 
[7] L. Vu, D. Chatterjee, and D. Liberzon, "Input-to-state stability of switched systems and switching adaptive control," Automatica, vol. 43, no. 4, pp. 639 - 646, 2007.

[8] J. P. Hespanha, D. Liberzon, and A. Teel, "Lyapunov conditions for input-to-state stability of impulsive systems," Automatica, vol. 44, pp. 2735 - 2744, 2008.

[9] R. Goebel, R. G. Sanfelice, and A. R. Teel, Hybrid Dynamical Systems: Modeling, Stability, and Robustness. Princeton University Press, 2012.

[10] C. Cai and A. Teel, "Characterizations of input-to-state stability for hybrid systems," Systems 83 Control Letters, vol. 58, no. 1, pp. 47-53, 2009.

[11] _ , "Robust input-to-state stability for hybrid systems," SIAM J. Control Optim., vol. 51, no. 2, pp. 1651-1678, 2013.

[12] N. Noroozi, A. Khayatian, and R. Geiselhart, "A characterization of integral input-to-state stability for hybrid systems," Math. Control, Signals, and Systems, vol. 29, no. 3, 2017.

[13] G. Zhang and A. Tanwani, "ISS Lyapunov functions for cascade switched systems and sampled-data control," Automatica, vol. 105, pp. 216-227, 2019.

[14] A. Chaillet, D. Angeli, and H. Ito, "Combining iISS and ISS with respect to small inputs: The strong iISS property," IEEE Trans. Automatic Control, vol. 59, no. 9, pp. 2518-2524, 2014.

[15] H. K. Khalil, Nonlinear Systems, 3rd ed. Prentice-Hall, Inc., 2002.

[16] D. Angeli, E. D. Sontag, and Y. Wang, "A characterization of integral input-to-state stability," IEEE Trans. Automatic Control, vol. 45, no. 6, pp. 1082-1097, 2000.

[17] A. Russo, S. Liu, D. Liberzon, and A. Cavallo, "Quasi-integral input-to-state stability for switched nonlinear systems," in proceedings of 21st IFAC World Congress, 2020.

[18] G. Yang and D. Liberzon, "A Lyapunov-based small-gain theorem for interconnected switched systems," Systems 8 Control Letters, vol. 78, pp. 47-54, 2015.

[19] S. Liu, A. Tanwani, and D. Liberzon. Average dwell-time bounds for ISS and integral ISS of switched systems using Lyapunov functions. [Online]. Available: http://liberzon.csl. illinois.edu/publications.html

[20] M. Arcak, D. Angeli, and E. Sontag, "A unifying integral ISS framework for stability of nonlinear cascades," SIAM J. Control Optim., vol. 40, no. 6, pp. 1888-1904, 2002. 